\title{
Prevalência de lesões bucais em crianças e adolescentes
}

\author{
Marina Aguiar Dias PEREIRA'; Linaena Méricy da SILVA²; Natália Galvão GARCIA ${ }^{3}$ \\ 1 - Aluna de graduação do curso de odontologia do Centro Universitário de Lavras (UNILAVRAS), Lavras-MG, Brasil; 2 - Doutora \\ em Patologia em consultório particular, Lavras-MG, Brasil; 3 - Doutora em Ciências Odontológicas Aplicadas, Professora do Curso \\ de Odontologia do Centro Universitário de Lavras (UNILAVRAS), Lavras - MG, Brasil.
}

\section{Resumo}

Objetivos: Avaliar a prevalência de lesões bucais em crianças e adolescentes em um centro odontológico universitário. Materiais e Métodos: Foram avaliados prontuários de pacientes entre 0 a 18 anos, atendidos no centro odontológico universitário no período de 2009 a 2018. Todas as lesões estomatológicas identificadas foram agrupadas de acordo com a sua natureza e pela localização anatômica. Os dados foram coletados e categorizados. Para quantificar a prevalência e verificar uma possível associação entre elas foi utilizado o teste de Qui-quadrado de Pearson. Resultados: Dos 17.000 prontuários de pacientes analisados, apenas 46 se encaixavam nos critérios de inclusão, nos quais, foram identificados 29 tipos de lesões, sendo a mucocele $(32,6 \%)$ a lesão de maior prevalência. A região mais acometida foi o lábio inferior, perfazendo $87 \%$ dos casos. Do total da amostra, o exame histopatológico foi realizado em apenas $24(52,2 \%)$ pacientes. Dentre esses, em $87 \%$ foi observada concordância entre o diagnóstico clínico e o histopatológico. Conclusões: Pode-se observar que a mucocele foi a lesão bucal mais prevalente nas crianças e adolescentes, sendo o lábio inferior, o local mais acometido. Desse modo, sugere-se que essa significativa prevalência da mucocele esteja diretamente associada à sua origem traumática, muito comum nessa faixa etária.

PALAVRAS-CHAVE: Mucocele; Criança; Adolescente; Diagnóstico Bucal.

AUTOR PARA CORRESPONDÊNCIA

Natália Galvão Garcia

Centro Universitário de Lavras (UNILAVRAS)

Rua Padre Jose Poggel, 506, Lavras-MG, CEP 3720.000 Brazil.

Telefone: +55 31 99403-3999 / E-mail: natggalvao@hotmail.com / nataliagalvao@unilavras.edu.br 


\section{Introdução}

A saúde bucal tem um papel fundamental na saúde geral das crianças, pois, problemas nutricionais, fonéticos e estéticos, podem ser decorrentes de alterações ou de patologias bucais. Desse modo, para a manutenção da saúde bucal adequada, é de grande importância que sejam realizadas ações de promoção de saúde, prevenção e diagnóstico das patologias bucais desde o nascimento ${ }^{1-3}$.

No entanto, a prática odontológica em pacientes pediátricos, nos últimos anos, tem se restringido aos cuidados com a preservação da dentição e dos tecidos periodontais, expressada basicamente pela prevenção e tratamento da cárie, doença periodontal e má-oclusão, marginalizando o diagnóstico e tratamento das outras patologias bucais que podem acometer esses pacientes ${ }^{2,4}$.

Entretanto, as patologias bucais envolvendo pacientes pediátricos apresentam uma prevalência significativa, sendo imprescindível que os cirurgiões-dentistas tenham conhecimento e estejam atentos às lesões bucais mais comumente encontradas, para que sejam realizadas ações de prevenção e promoção de saúde voltada para esses aspectos ${ }^{3,5}$.

Desse modo, esse trabalho teve como objetivo avaliar a prevalência de lesões bucais em crianças e adolescentes em um centro odontológico universitário.

\section{Métodos}

Este trabalho foi submetido à apreciação do Comitê de Ética em Pesquisa em Seres Humanos do Centro Universitário de Lavras e aprovado (CAAE: 87236218.1.0000.5116).

Para realização deste estudo retrospectivo foi feita a análise de 17.000 de prontuários de pacientes que receberam atendimentos nas Clínicas de Odontologia do Centro Universitário de Lavras - MG, no período de 2009 a 2018. 
As avaliações foram feitas por três avaliadores cegos. Todas as alterações estomatológicas encontradas dos pacientes que se enquadravam nos seguintes critérios de inclusão: prontuários de pacientes assinados pelos pais ou responsáveis; e prontuários de pacientes entre zero e 18 anos de idade. Como critérios de exclusão foram utilizados: prontuários incompletos, com ausência de dados clínicos e histopatológicos.

As lesões foram agrupadas de acordo com a sua natureza e pela localização anatômica: língua, lábio inferior, mucosa jugal, assoalho bucal, palato duro e mole, etc.

Dados clínicos, como sexo, idade, raça, localização anatômica, diagnóstico clínico e histopatológico foram coletados e categorizados. As variáveis definidas no estudo foram organizadas em planilhas do programa Excel ${ }^{\circledR}$ e para quantificar a prevalência e verificar uma possível associação entre elas foi utilizado o teste de Qui-quadrado de Pearson, considerando o nível de significância de $5 \%(p<0,005)$.

\section{Resultados}

Dos 17.000 prontuários de pacientes analisados, apenas 46 se encaixavam nos critérios de inclusão, não sendo observada diferença de acometimento entre os sexos, sendo 50\% do sexo feminino e $50 \%$ do sexo masculino. A idade média dos pacientes selecionados foi 11,3 anos $( \pm 4,6)$, sendo a idade mínima 2 anos e a máxima 18 anos.

Quanto à cor da pele dos pacientes, 44\% deles relataram ser leucodermas, $26 \%$ feodermas, $13 \%$ melanodermas e em $17 \%$ dos prontuários não havia esta informação.

Nos 46 prontuários analisados, foram identificados 29 tipos de lesões, as quais foram agrupadas de acordo com a sua natureza nos seguintes grupos: lesões de glândulas salivares; lesões reacionais; lesões infecciosas; lesões pigmentadas; variações da normalidade; cistos e pseudocistos; tumores benignos 
e malignos. Sendo a mucocele, a lesão de maior prevalência observada em $32,6 \%$ dos casos, como descrito na Tabela 1.

A região mais acometida foi o lábio inferior, perfazendo $87 \%$ ( $p=$ $0,04)$ dos casos, seguido pela língua $(4,4 \%)$, mucosa jugal $(4,3 \%)$ e mandíbula $(4,3 \%)$ (Gráfico 1$)$.

Do total da amostra, o exame histopatológico foi realizado em apenas $24(52,2 \%)$ pacientes. Dentre eles, a mucocele também se apresentou como a lesão de maior prevalência ( $58,3 \%)$, conforme mostra Tabela 2.

Dentre os prontuários ( $\mathrm{n}=24)$ que apresentavam o exame histopatológico, 23 tinham a hipótese clínica e o diagnóstico histopatológico propriamente dito. Dentre esses 23, em 20 (87\%) foi observada concordância entre o diagnóstico clínico e o histopatológico (Gráfico 2).

GRÁFICO 1 · Frequência da localização das lesões diagnosticadas.
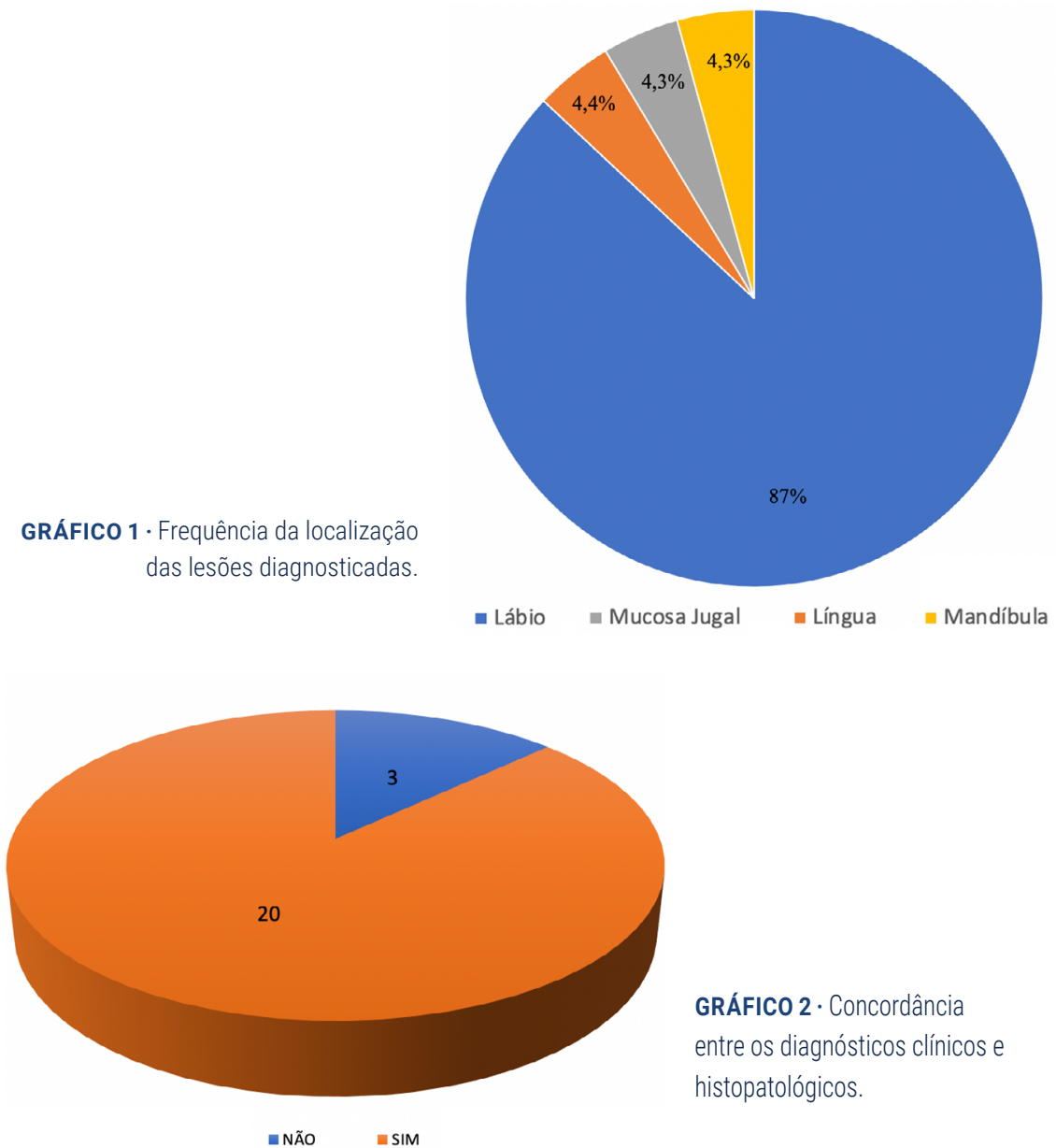

GRÁFICO 2 - Concordância entre os diagnósticos clínicos e histopatológicos. 
TABELA $1 \cdot$ Prevalência das lesões diagnosticadas clinicamente

\begin{tabular}{|c|c|c|c|}
\hline Grupos & Diagnóstico clínico & Prevalência & IC (95\%) \\
\hline \multirow{3}{*}{$\begin{array}{l}\text { Lesões de glândulas } \\
\text { salivares }\end{array}$} & Mucocele & $32,6 \%(n=14)$ & $0,2502-0,4965$ \\
\hline & Rânula & $4,3 \%(n=2)$ & $0,0055-0,1200$ \\
\hline & Sialolitíase & $2,1 \%(n=1)$ & $0,0008-0,0968$ \\
\hline \multirow{4}{*}{ Lesões reacionais } & Hiperplasia Fibrosa Inflamatória & $6,5 \%(n=3)$ & $0,0124-0,1417$ \\
\hline & Granuloma Piogênico & \multirow{3}{*}{$2,1 \%(n=1)$} & \multirow{3}{*}{$0,0008-0,0968$} \\
\hline & Hiperplasia Epitelial Focal & & \\
\hline & Lesão Periférica de Células Gigantes & & \\
\hline \multirow{3}{*}{ Lesões infecciosas } & Papiloma & $4,3 \%(n=2)$ & $0,0055-0,1200$ \\
\hline & Gengivoestomatite Herpética & \multirow{2}{*}{$2,1 \%(n=1)$} & \multirow{2}{*}{$0,0008-0,0968$} \\
\hline & Molusco contagioso & & \\
\hline \multirow{3}{*}{ Lesões Pigmentadas } & Melanose & \multirow{3}{*}{$2,1 \%(n=1)$} & \multirow{3}{*}{$0,0008-0,0968$} \\
\hline & & & \\
\hline & Nevus melanocítico & & \\
\hline \multirow{4}{*}{$\begin{array}{l}\text { Variações da } \\
\text { normalidade }\end{array}$} & Língua Geográfica & \multirow{4}{*}{$2,1 \%(n=1)$} & \multirow{4}{*}{$0,0008-0,0968$} \\
\hline & Linha Alba & & \\
\hline & Má formação vascular & & \\
\hline & Displasia Ectodérmica & & \\
\hline \multirow{6}{*}{ Cistos e pseudocistos } & Cisto Odontogênico Calcificante & \multirow{6}{*}{$2,1 \%(n=1)$} & \multirow{6}{*}{$0,0008-0,0968$} \\
\hline & Cisto do Desenvolvimento & & \\
\hline & Cisto dentígero & & \\
\hline & Cisto Periodontal Lateral & & \\
\hline & Queratocisto Odontogênico & & \\
\hline & Cisto Ósseo Simples & & \\
\hline \multirow{6}{*}{$\begin{array}{l}\text { Tumores benignos e } \\
\text { malignos }\end{array}$} & Odontoma & \multirow{6}{*}{$2,1 \%(n=1)$} & \multirow{6}{*}{$0,0008-0,0968$} \\
\hline & Tumor Odontogênico Adenomatóide & & \\
\hline & Neurofibroma & & \\
\hline & Lipoma & & \\
\hline & Linfangioma & & \\
\hline & Adenocarcinoma & & \\
\hline
\end{tabular}

Fonte: Dados da pesquisa (2019) 


\section{Discussão}

O levantamento das lesões da mucosa bucal e do complexo maxilomandibular em crianças e adolescentes caracteriza um estudo dentro de uma população específica ${ }^{6,7}$. No presente trabalho, consideramos como idade de referência, a faixa etária compreendida entre zero a dezoito anos de idade. Pois, convencionalmente é esta a faixa etária delimitada pelo Estatuto da Criança e do Adolescente. Além disso, seguindo essa faixa etária haveria a abrangência de toda a fase da dentição decídua e mista, chegando até a permanente, mesmo que haja algum atraso relativo à normalidade, de caráter local ou sistêmico.

No presente estudo foram encontrados 29 tipos de afecções, sendo a mucocele, a mais prevalente. A qual trata-se de uma lesão não infecciosa benigna que se desenvolve em mucosa oral, geralmente resultante da ruptura do ducto de glândulas salivares menores ou da presença de cálculos (sialolitos) que impedem a drenagem do muco através do ducto excretor, provocando a dispersão de mucina para o interior do tecido conjuntivo ${ }^{5}$. Estudos semelhantes relatados na literatura, ao avaliarem lesões orais

TABELA 2 Prevalência das lesões diagnosticadas histopatologicamente

\begin{tabular}{|c|c|c|c|}
\hline Grupos & Diagnóstico Histopatológico & Frequência & IC (95\%) \\
\hline $\begin{array}{l}\text { Lesões de glândulas } \\
\text { salivares }\end{array}$ & Mucocele & $58,3 \%(n=14)$ & $0,43-0,82$ \\
\hline Lesões reacionais & $\begin{array}{l}\text { Hiperplasia Fibrosa Inflamatória } \\
\text { Granuloma Piogênico }\end{array}$ & $4,17 \%(n=1)$ & $0,00-0,12$ \\
\hline Lesões infecciosas & Papiloma & $4,17 \%(n=1)$ & $0,00-0,12$ \\
\hline $\begin{array}{l}\text { Variações da } \\
\text { normalidade }\end{array}$ & $\begin{array}{l}\text { Displasia óssea } \\
\text { Língua geográfica }\end{array}$ & $4,17 \%(n=1)$ & $0,00-0,12$ \\
\hline Cistos e pseudocistos & $\begin{array}{l}\text { Cisto radicular } \\
\text { Cisto dentígero } \\
\text { Cisto Ósseo }\end{array}$ & $4,17 \%(n=1)$ & $0,00-0,12$ \\
\hline $\begin{array}{l}\text { Tumores benignos e } \\
\text { malignos }\end{array}$ & $\begin{array}{l}\text { Odontoma } \\
\text { Fibroma ossificante periférico }\end{array}$ & $4,17 \%(n=1)$ & $0,00-0,12$ \\
\hline
\end{tabular}

Fonte: Dados da pesquisa (2019). 
em pacientes na mesma faixa etária também ressaltaram ter encontrado a mucocele como a lesão de maior incidência ${ }^{6,8-10}$. A localização mais acometida identificada foi o lábio inferior, indo de encontro aos demais estudos epidemiológicos publicados ${ }^{9-13}$.

Embora o diagnóstico clínico das mucoceles, na maioria das vezes, não ofereça dificuldades ao profissional, é comum sua semelhança com outras lesões, como fibroma, lipoma, granuloma piogênico, que também podem acometer a cavidade oral e pode gerar uma divergência entre o resultado clínico e o histopatológico ${ }^{14,15}$. No presente estudo foi identificada uma concordância entre o diagnóstico clínico e o histopatológico em $87 \%$ dos casos, no entanto, o exame histopatológico foi realizado em apenas $24(52,2 \%)$ prontuários.

\section{Conclusão}

Com base na amostra analisada pode-se observar que a mucocele foi a lesão bucal mais prevalente nas crianças e adolescentes, sendo o lábio inferior, o local mais acometido. Desse modo, sugere-se que essa significativa prevalência da mucocele esteja diretamente associada à sua origem traumática, muito comum nessa faixa etária. Além disso, esse estudo ressalta a variedade de lesões estomatológicas que podem envolver crianças e adolescentes, sendo de grande importância que o cirurgião-dentista saiba identificar e diagnosticar essas lesões, contribuindo para o bem-estar desses pacientes.

\section{Referências}

1 - More CB, Bhavsar K, Varma S, Tailor M. Oral mucocele: a clinical and histopathological study. J Oral Maxillofac Pathol. 2014; 18(Suppl 1): S72-7.

2 - Wang YL, Chang HH, Chang JY, Huang GF, Guo MK. Retrospective survey of biopsied oral lesions in pediatric patients. J Formos Med Assoc. 2009; 108(11): 862-71.

3 - Kwok EY, Dovigi EA, Eversole LR, Dovigi AJ. Pediatric oral pathology: a retrospective survey of 4,554 biopsies. Pediatr Dent. 2015; 37(7): 546-9. 
4 - Hipolito RA, Martins CR. Prevalência de alterações da mucosa bucal em adolescentes brasileiros institucionalizados em dois centros de reeducação. Ciênc saúde coletiva. 2010; 15(2): 3233-3242.

5 - Valério RA, Queiroz AM, Romualdo PC, Brentegani LG, PaulaSilva FW. Mucocele and fibroma: treatment and clinical features for differential diagnosis. Brazilian Dental Journal. 2013; 24(5): 537-541.

6 - Lima GS, Fontes ST, Araújo LM, Etges A, Tarquinio SB, Gomes AP. A survey of oral and maxillofacial biopsies in children: a single-center retrospective study of 20 years in Pelotas-Brazil. J Appl Oral Sci. 2008; 16(6): 397-402.

7 - Sousa FB, Etges A, Corrêa L, Mesquita RA, Araújo N. Pediatric oral lesions: a 15-year review from São Paulo, Brazil. J Clin Pediatr Dent. 2002; 26(4): 413-8.

8 - Chen YK, Lin LM, Huang HC, Lin CC, Yan YH. A retrospective study of oral and maxillofacial biopsy lesions in a pediatric population from southern Taiwan. Pediatr Dent. 1998; 20(7): 404-10.

9- Re CD, Achilli A, Tarozzi M, Lodi G, Demarosi F, Sardella A, Carrassi A. Mucoceles of the oral cavity: a large case series (1994-2008) and a literature review. Med Oral Patol Oral Cir Bucal. 2010; 15(4): e551-6.

10 - Cavalcante RB, Turatti E, Daniel AP, Alencar GF, Chen Z. Retrospective review of oral and maxillofacial pathology in a Brazilian paediatric population. Arch Paediatr Dent. 2016; 17(2): 115-22.

11 - Jones AV, Franklin CD. An analysis of oral and maxillofacial pathology found in children over a 30-year period. Int J Paediatr Dent. 2006; 16(1): 19-30.

12 - Bezerra TM, Monteiro BV, Henriques ÁC, Vasconcelos CM, Nonaka CF, Costa MMC. Epidemiological survey of mucus extravasation phenomenon at an oral pathology referral center during a 43 year period. Braz J Otorhinolaryngol. 2016; 82(5): 536-42.

13 - Silva LVO, Arruda JAA, Martelli SJ, Kato CNAO, Nunes LFM, Vasconcelos ACU, et al. A multicenter study of biopsied oral and maxillofacial lesions in a Brazilian pediatric population. Braz Oral Res. 2018; 32(e20): 1-9.

14 - Shah SK, Le MC, Carpenter WM. Retrospective review of pediatric oral lesions from a dental school biopsy service. Pediatr Dent. 2009; 31(1): 14-9.

15 - Das S, Das AK. A review of pediatric oral biopsies from a surgical pathology service in a dental school. Pediatr Dent. 1993; 15(3): 208-11. 


\title{
Prevalence of oral lesions in children and adolescents
}

\begin{abstract}
Objectives: To evaluate the prevalence of oral lesions in children and adolescents at university dental center. Matherials and Methods: Medical records of patients between 0 and 18 years old, seen at the university dental center from 2009 to 2018, were evaluated. All identified dental lesions were grouped according to their nature and anatomical location. The data were collected and categorized. To quantify the prevalence and verify a possible association between them, Pearson's chi-square test was used. Results: Of the 17,000 medical records of patients analyzed, only 46 met the inclusion criteria, in which 29 types of lesions were identified, with mucocele (32.6\%) being the most prevalent lesion. The most affected region was the lower lip, making up $87 \%$ of the cases. Of the total sample, histopathological examination was performed in only 24 (52.2\%) patients. Among these, in $87 \%$ there was an agreement between clinical and histopathological diagnosis. Conclusions: It can be seen that the mucocele was the most prevalent oral lesion in children and adolescents, with the lower lip being the most affected site. Thus, it is suggested that this significant prevalence of the mucocele is directly associated with its traumatic origin, very common in this age group.
\end{abstract}

KEYWORDS: Mucocele; Child; Adolescent; Diagnosis oral.

\section{Como citar este artigo}

Pereira MAD, Silva LM, Garcia NG. Prevalência de lesões bucais em crianças e adolescentes. Rev Odontol Bras Central 2021; 30(89): 162-170. DOI: 10.36065/robrac.v30i89.1493 